Bull. Mater. Sci., Vol. 4, No. 5, December 1982, pp. 549-561.

(C) Printed in India.

\title{
Melt-spinning technique for preparation of metallic glasses
}

\author{
R C BUDHANI, T C GOEL and K L CHOPRA \\ Department of Physics, Indian Institute of Technology, New Delhi 110016, India.
}

MS received 12 December 1981

\begin{abstract}
Rapid solidification of metallic melts at a rate $\subsetneq 10^{5} \mathrm{~K} / \mathrm{sec}$ has gained considerable interest for casting non-equlibrium crystalline structures in general and metallic glasses in particular. Of the variety of techniques used for rapid solidification, melt-spinning and melt-extraction are widely used. This paper describes the design and development of a melt-spinning technique in our labaratory. Optimization of spinning parameters for smooth and continuous ribbons is discussed. Glassy ribhons of $\mathrm{Cu}-\mathrm{Zr}$ and $\mathrm{Pd}-\mathrm{Ge}$ alloys have been mude under optimized conditions. A brief account of some of the structural and electronic properties of these glasses is illustrated.
\end{abstract}

Keywords. Amarphous miterials; electron transport; melt-spinning; metallic glasses; photoemission; rapid solidification.

\section{Introduction}

The familiar glasses are substances which are transparent to light, colourless, brittle and bad conductors of electricity and heat. Technologically, such a glass is an inorganic product of fusion, cooled to a rigid condition without crystallizing . Metals, on the other hand, are opaque, ductile, lustrous and good conductors of heat and electricity. Depending upon composition and technique, melts of some metals with other metals and non-metals can be cooled to rigid conditions avoiding crystallization. Such materials are typically metallic though structurally glassy (non-crystalline) and are therefore known as metallic glasses.

The absence of long-range periodicity in these materials brings about significant changes in their mechanical, chemical and physical properties which has opened new vistas of such technological applications as magnetic device fabrication (Lubrosky et al 1978), high saturation current superconductors (Johnson and Williams 1979), high strength engineering materials (Cahn 1980), corrosion resistance and tribological uses (Naka et al 1978). Moreover, these materials have mobilized the world of basic science in order to examine the thermodynamic aspects of glass formation, stability of the glassy phase under different thermodynamic conditions and structural and morphological changes during the transformation process. Topological and electronic band structural modifications brought about by the rapid solidification process and their influence on electron 
transport processes are exciting fields of research. Magnetic properties, under dilute and concentrated impurity regimes are being studied to investigate the influence of disorder on local moment formation and coupling of impurity moments. Emergence of a host of surface analysis techniques has accelerated the efforts for probing the electronic band structure, short-range order and its topology, diffusion and relaxation processes.

A variety of techniques for rapid solidification at a rate exceeding a critical cooling rate for glass formation have emerged and a number of glassy alloys have been made (Suryanarayana 1981). In $\S 2$ of this paper, we briefly review the techniques being used for rapid solidification. $\S 3$ deals with the design and development of a melt-spinning technique in our laboratory. In $\S 4$ the main features concerning the formation, structural stability, electron transport properties and electronic structure of $\mathrm{Pd}-\mathrm{Ge}$ and $\mathrm{Cu}-\mathrm{Zr}$ glasses are described.

\section{Techniques for rapid solidification}

In order that a material be vitrified by continuous cooling trom its molten state, cooling must be sufficiently rapid through the equilibrium melting point such that crystallization can be avoided. Under such a condition, the viscosity increases and the configurational entropy decreases continuously till the material reaches its glass transition temperature, characterised by a viscosity, $\eta \approx 10^{13}$ poise (Turnbull 1969). For metallic melts, since the viscosity at melting point is very low, solidification of the liquid-like structure requires a sufficiently high cooling rate $\left(\sim 10^{5} \mathrm{~K} / \mathrm{sec}\right)$ (Davis 1978). To achieve cooling rates of this magnitude, all rapid quenching techniques are based on the principle of bringing the melt in contact with a highly conducting surface/surfaces of relatively large heat capacity at high velocity such that thin sections of the melt come in intimate contact with it. To meet the requirement of optimum cooling rate, uniformity, shape, quantity and reproduciblity of the samples prepared and to cover high melting temperature and reactive alloys, a veriety of techniques have come up. Table 1 lists the main techniques and their characteristic features.

As seen from the table, all single foil techniques have a fairly high cooling ra te but the small amount of material formed and its shape limit their utility to academic curiosity only. Furthermore, particularly in the 'piston-and-anvil' technique, significant amount of stresses are introduced in the samples which may yield properties different from those of a freely grown non-crystalline structure. However, gun-technique needs a specific mention. Akhtar et al (1979a) in a detailed investigation of quenching parameters such as gun rupture pressure, vacuum and gaseous environment, have established that better vacuum conditions and high rupture pressure help in enhancing the cooling rates by at least two orders of magnitude. Such high cooling rates and greater manoeuverability over the process parameters lead to metastable and non-crystalline phaseformation in more number of alloys.

The utility of thick film techniques, such as plasma spray, is limited to applications where a surface has to be protected from wear and corrosion. Double roller technique has its own limitation as regards the costing of smooth and 


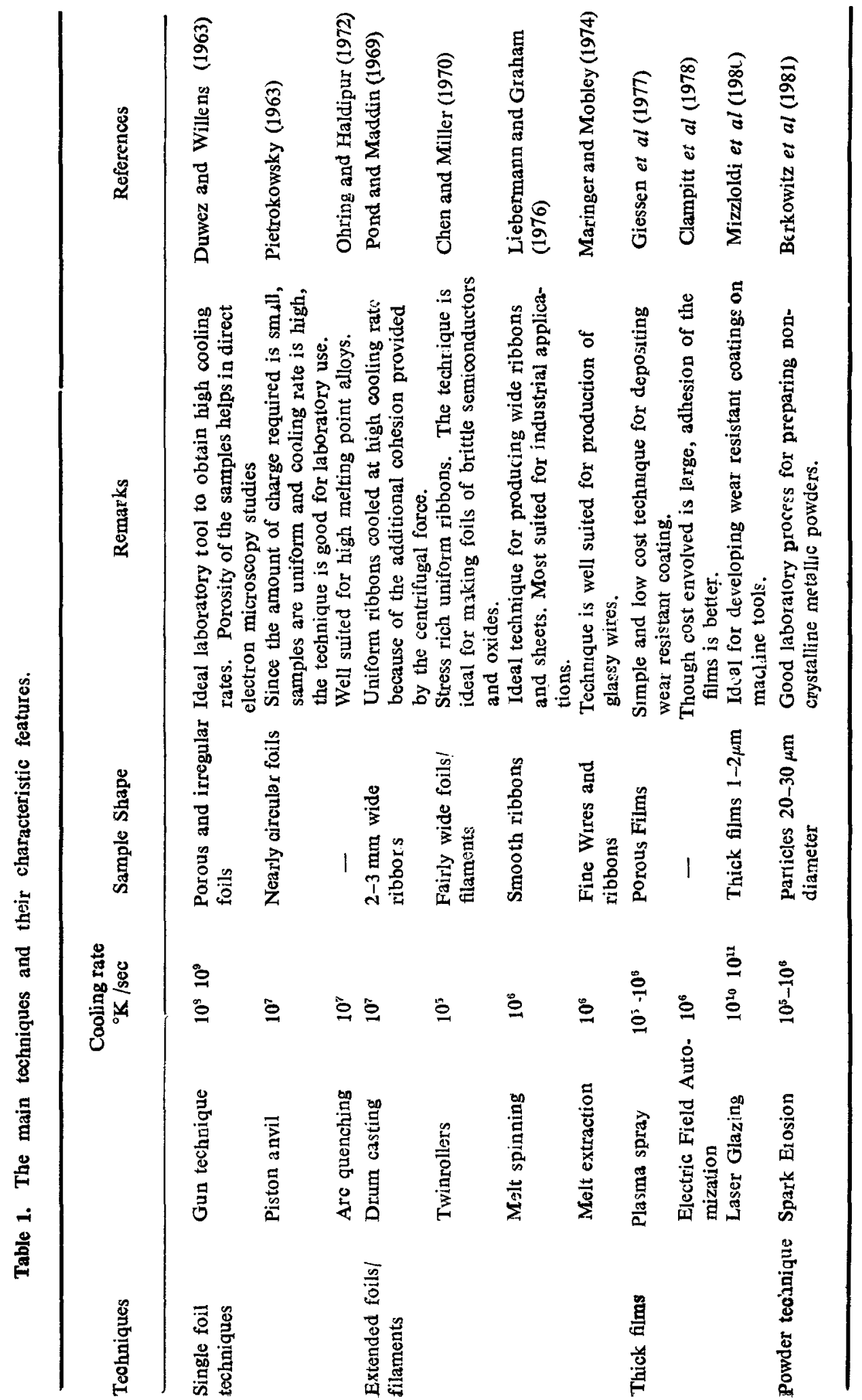


continuous filaments of glassy and metastable crystalline structures. Because of the short melt substrate contact time, the cooling rate achieved is not enough to cover many useful glass-forming systems. Besides, the squeezing of the material between the nip of two rollers leads to formation of parabolic stress zones on the ribbon surface.

Melt-spinning and melt-extraction techniques are widely used as they do not suffer from the limitations of the other rapid solidification techniques. However, it is to be noted that in melt-extraction, a large melt bath is required which leads to oxidation of the melt surface and heating of the wheel. In a melt-spinning technique, the melt is far from the roller surface and th us heating of the wheel is minimized. The ribbon width can be increased using a linear configuration of nozzles or by a rectangular slit. Layered structures of two or more materials can also be made by coupling sequential nozzles. Further, the molten material remains in a closed crucible so that oxidation of the melt is avoided even if the operation is carried out in air. Moreover, in melt-spinning, a better control over the ribbon dimensions and cooling rate can be achieved with a proper control on the melt pressure, nozzle inclination and nozzle diameter.

\section{Melt-spinning technique}

\subsection{Design and fabrication}

The set-up shown in figures $1 \mathrm{a}$ and $\mathrm{b}$ essentially consists of three parts, (a) furnace-crucible-pressure transfer assembly, (b) high thermal inertia rotating substrate combined with a quench stabilizer and a wiper and (c) the vacuum system. A schematic diagram of part (a) and two vertical sections, rotated through $90^{\circ}$ of part (b) are shown in figure 2 .

The furnace-crucible-pressure transfer assembly consists of a pressure line $C$ one end of which is connected to a flexible bellow which passes through the base plate via an O-ring seal and is connected to argon gas reservoir. The other end of line $\mathrm{C}$ is vacuum tight with the crucible holder $E$. A crucible $(F)$, made of fused or transparent silica with a nozzle at the bottom is placed on the seating (D) made of neoprene O-rings. The thermal inertia of part $E$ is high so that the heat conducted from the hot end of the crucible is quickly dissipated without causing any damage to the seat. To melt the material, the lower portion of the crucible is heated with a resistance furnance $(G)$ which consists of Kanthal/tungsten spiral $(H)$. The spiral is surrounded by a fused silica cylinder $(I)$ which acts as thermal and electrical shield between the spiral and stainless steel capsule $(J)$, which contains both. The free ends of the spiral are taken out and connected to two sliding brass blocks $M_{1}$ and $M_{2}$. $M_{1}$, which is electrically isolated from the supporting rod $(L)$, is connected to power mains and $M_{2}$ is grounded. A chromel-alumel thermocouple has been incorporated in the furnace to monitor the temperature during heating. Part $K$ of the arrangement, connected to a moving platform on the base plate provides for vertical, lateral and tilt adjustments. A micromotion arrangement, consisting of $A, B$ and $C$ has been incorporated in the cavity of $K$. On rotating $A$, depending on the direction of rotation, the pressure line $C$ along with the crucible holder, crucible and the furnace 
can be moved up and down very precisely. The substrate part (b) is made of $17.5 \mathrm{~cm}$ diameter mild steel wheel on periphery of which a $5 \mathrm{~mm}$ thick electrolytic copper rim is thorougly welded. The roller, with its shaft held on two mild steel blocks, is rotated up to $6000 \mathrm{rpm}$. A polishing arrangement $P$ has been incorporated to clean the roller surface during each rotation. To avoid oxidation of the melt and to study the influence of gaseous environment at crucible-substrate spacing, a rectangular slit type nozzle is placed just above the wiper to provide desired gaseous environment. The whole assembly is contained in a cylindrical steel chamber vacuum sealed to the base plate with a neoprene O-ring. The chamber is connected to a conventional oil diffusing pump vacuum system and can be evacuated down to $\sim 10^{-4}$ Torr.

\subsection{Working principle}

A simple representation of the working of the technique is shown in figure 3. The molten material is driven from the nozzle in the form of a jet, which on impingement with the rotating disc forms a melt puddle. The shape of the puddle is formed under the counteracting forces of tangential flow of the material and the surface tension. Because of the low viscosity of the molten alloy/metal, the shear or vorticity layers extend only a few microns from the surface of the roller into the puddle and it stays on the roller surface. Because of a large temperature difference at the melt-substrate interface, the melt beneath the puddle solidifies into a ribbon. The ribbon remains in contact with the disc surface up to $10^{\circ}$ arc and then leaves it under the action of the centrifugal force. However, the fabrication of continuous ribbons quenched under optimum cooling rates is not simple. A prior knowledge of the jet dynamics (Cline and Anthony 1978), stability of the puddle (Walter and Luborsky 1977) (which is greatly

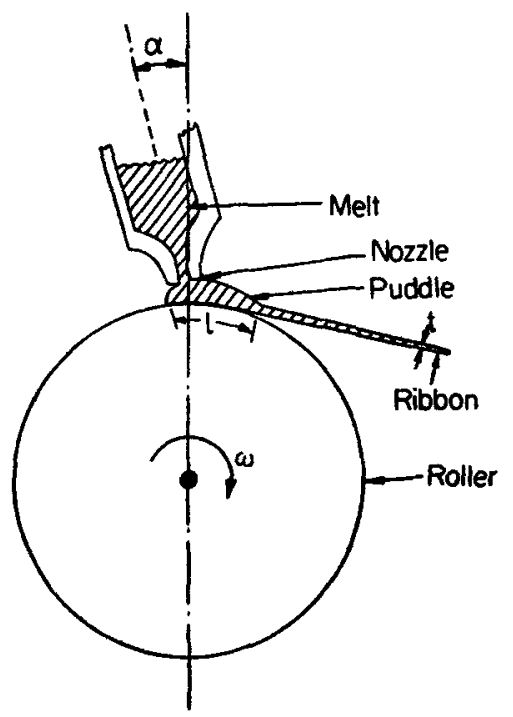

Figure 3. A schematic diagram of the shape of the puddle and consequent ribbon formation when the molten metal/alloy touches the surface of the rotating wheel, 
affected by vibrations) and optimization of parameters like ejection pressure $p$, nozzle diameter $\phi$ and surface speed $V_{s}$ of the wheel are required. A detailed analysis of the ribbon dimensions and uniformity has been discussed by Anthony and Cline (1979), a brief account of the process parameters and their optimization relevant to the formation of ribbons with a typical case of $\mathrm{Pb}-\mathrm{Sn}$ alloys is given below.

\subsection{Ribbon dimensions}

Assuming that a molten metal of density $\rho_{l}$ coming out from a nozzle of diameter $\phi$ at a speed $V_{\phi}$ solidifies to a ribbon of cross-sectional area $A_{r}$ and density $\rho_{r}$, then the continuity of the ribbon demands that the mass of the ribbon formed in unit time should equal the jet flow rate (Liebermann and Graham 1976), i.e.,

$$
\begin{aligned}
& \frac{1}{4} \pi \phi^{2} V_{\phi} \rho_{l}=A_{r} V_{s} \rho_{r} \\
& A_{r}=\frac{1}{4} \pi\left(\frac{\rho_{l}}{\rho_{r}}\right) \phi^{2} \frac{V_{\phi}}{V_{s}} .
\end{aligned}
$$

From this equation it can be easily seen that the area of cross-section for a given material is a function of $\phi, V_{\phi}$ and $V_{s}$. Moreover, apart from jet dimensions, the thickness of the ribbon is governed by detailed shape of the puddle, jet inclination $a$ and the mode of heat transfer at melt roller interface. For an ideal contact with no temperature discontinuity at the interface, the thickness $h$ of the ribbon will increase with the dwell time $\tau\left(=l / V_{s}\right)$ as

$$
h=2(D \tau)^{1 / 2}=2\left(l / V_{s}\right)^{1 / 2} \cdot D^{1 / 2},
$$

where $D$ is the thermal diffusivity. If the thermal contact between the material and the substrate is poor, the temperature gradient inside the puddle is negligible and the heat transfer is primarily interface-controlled. This situation corresponds to Newtonian cooling (Ruhl 1967). If the contact is poor and the coefficient of Newtonian cooling is taken as constant, independent of time and position at the ribbon-roller interface, the ribbon thickness is given by the expression (Anthony and Cline 1979),

$$
h \propto(\triangle T / \triangle H),
$$

where $\triangle T$ is the temperature difference between opposite sides of the interface, and $\Delta H$ is the heat of solidification per unit volume of the melt. However, in actual practice, an intermediate situation is obtained and 1 ts closeness to the ideal condition is determined by various factors like temperature of the melt, position within the puddle and melt-substrate material. The physical condition of the substrate is equally important because it determines the degree of intimate contact between the melt and the substrate. On a polished, highly conducting surface, the ribbon remained in contact for a sufficiently long time. Therefore at least beneath the puddle, cooling can be taken to the ideal. Under this condition, the variation of the thickness of the ribbons is determined by (2). Assuming a constant puddle length $l$ (or, in other words, for a given nozzle diameter, nozzle pressure and $a$ ), the thickness is inversely proportional to the square root of the rotation speed $\left(h \propto V_{3}^{-1 / 2}\right)$. For a given speed $V_{3}$ the ribbon thickness is directly 
proportional to square root of puddle length $\left(h \propto l^{1 / 2}\right)$. The thickness variation of the $\mathrm{Pb}-\mathrm{Sn}$ eutectic alloy as a function of $V_{s}$ and $a$ with a given $\phi$ is shown in figure $4 \mathrm{a}, \mathrm{b}$. During all the runs, the temperature of the melt was kept $100^{\circ} \mathrm{C}$ above the eutectic point. Figure 4 clearly illustrates that the thickness increase $s$ with increasing $a$ and decreasing $V_{c}$. However, $\alpha$ cannot be increased indefinitely because, beyond a certain value, the melt under the joint influence of initial speed and the centrifugal force tries to fly away from the wheel without wetting it. The functional dependence of $\alpha$ and $V_{s}$ follows the predictions of ideal cooling theory. The lowest and highest permissible values of $V_{s}$ for $a=15^{\circ}$ are $\simeq 10 \mathrm{~m} / \mathrm{sec}$ and $\simeq 57 \mathrm{~m} / \mathrm{sec}$. If $V_{s}$ is lower or higher than these values, the continuity equation at the nozzle-roller contact point is not satisfied and the melt forms globules in the former and broken ribbons in the latter case.
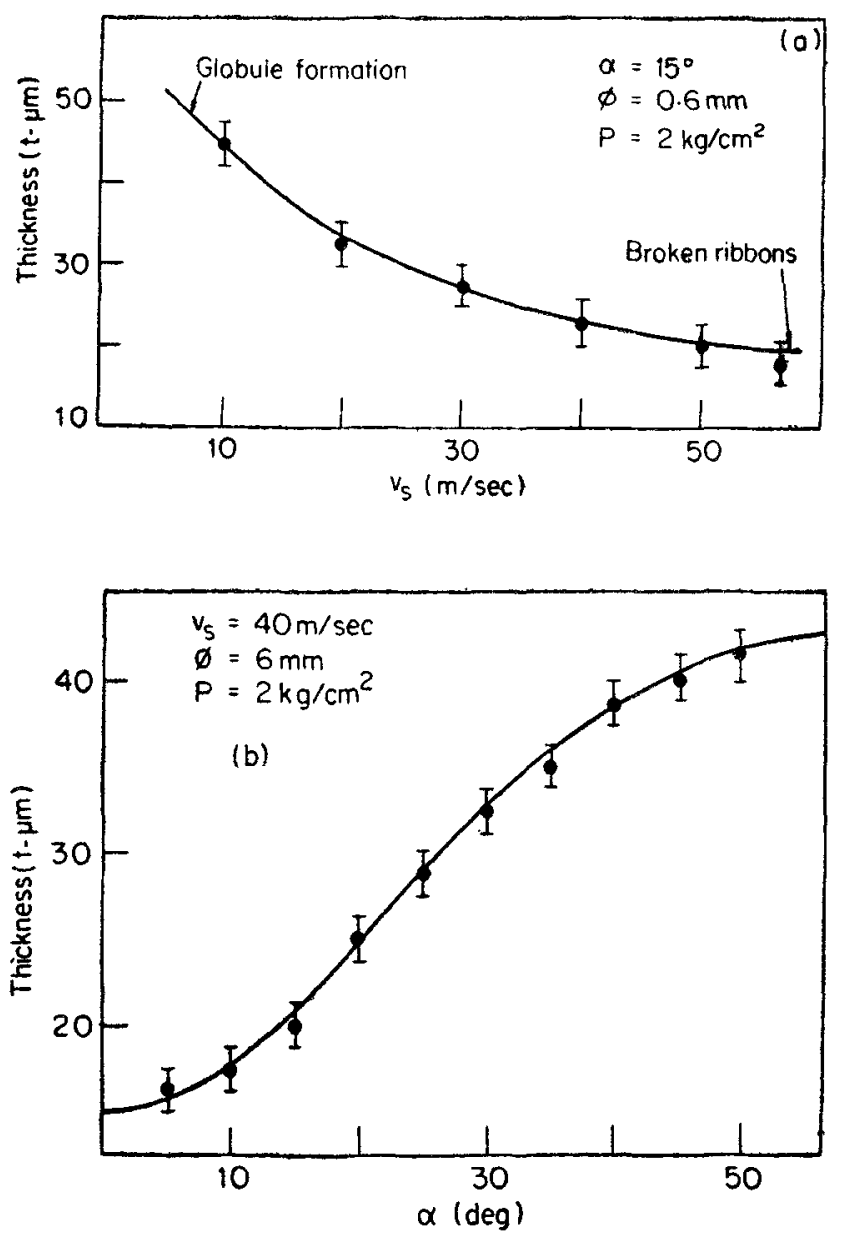

Figure 4. Variation of the ribbon thickness, (a) as a function of the roller speed $V_{s}$ for given values of ejection pressure $p$, nozzle diameter $\phi$ and nozzle angle $a$ and (b) as a function of $a$ when $V_{s}, p$ and $\phi$ are constant. 


\subsection{Dimensional variations}

The above description gives the main factors responsible for the width and the thickness of ribbons. Other factors, like the temperature of the melt, gaseous environment, jet geometry and puddle vibrations also influence the thickness and the width. Anthony and Cline (1979), through a detailed mathematical analysis of the dynamics of the melt from jet to puddle formation, have shown that jet instability and turbulance, which arises because of the variation in the nozzle design liquid head in the crucible and the deriving pressure can lead to a velocity gradient along the segments in the cross-section. The puddle formed as a consequence is dynamically unstable and therefore leads to ribbon non-uniformity. Even in the case of an ideal jet, oscillations in the puddle along the direction of extraction and along the width can contribute to thickness variations. However, these effects occur with low frequency and are not detectable with naked eye. These effects become significant when natural modes of melt resonate with the vibrations associated with a dynamically unstable wheel and imperfections on the wheel surface. Thickness variations along the width are more sensitive to the melt temperature. In our experiments, since the liquid head varies with time (because of the limited amount of charge taken at the beginning), dimensional variations do occur along the length of the ribbons, in particular, at the later stage of ribbon formation when liquid in the column is about to deplete. For $V_{s} \simeq 40 \mathrm{~m} / \mathrm{sec}$ and $a=15^{\circ}$, the ribbons are fairly smooth. Our telestep and scanning electron microscopy studies on ribbons prepared under different quenching conditions revealed that smoothness of the top surface, which does not come in contact with the wheel, is highly sensitive to the melt temperature. On increasing the melt temperature to $300^{\circ} \mathrm{C}$ above the melting point of the alloy, the top surface of the ribbons shows large thickness variations. The roughened top surface as a consequence of the increased melt temperature is probably because of a drastic decrease in the viscosity resulting thereby in wave formation on the puddle top. Topology of the contact face, on the other hand, is determined by the particle size of the abrasive used for polishing.

\section{Formation of metallic glasses}

Glassy ribbons of copper-zirconium alloys in the composition range 33.5 to $72 \%$ copper and palladium-germanium alloys in the range from 20 to $22.5 \%$ Ge have been made in our laboratory. Extended range of glass formation in $\mathrm{Cu}-\mathrm{Zr}$ system covers four equilibrium phases, namely, $\mathrm{Cu}_{8} \mathrm{Zr}_{3}, \mathrm{Cu}_{10} \mathrm{Zr}_{7}, \mathrm{CuZr}$ and $\mathrm{CuZr}_{2}$. A comparative study of glass formation (Budhani et al 1981a) in these alloys with melt-spinning and gun-quenching techniques, which have cooling rates different by at least two orders of magnitude, reveals that with gun-quenching the glassy phase can be retained up to 80 atomic per cent $\mathrm{Zr}$. High cooling rate associated with the gun technique, however, did not extend the glassy phase towards the $\mathrm{Cu}$ rich side of the equilibrium phase diagram. The abrupt change at $72 \% \mathrm{Cu}$ with both the techniques is primarily due to the high stability of the intermetallic compound $\mathrm{Cu}_{3 \cdot 8} \mathrm{Zr}$ (varying stoichiometry $\mathrm{Cu}_{4} \mathrm{Zr}$ to $\mathrm{Cu}_{3} \mathrm{Zr}$ ). Glass formation in the range of four equilibrium intermetallic compounds is due to their relative 
instability which is reflected in terms of the loose structures and low melting points and a mutual competition between the phases to nucleate on cooling from the melt. Thermal analysis and activation energy calculations from isochronel annealing behaviour of resistivity show increasing stability of the glass with copper concentration. The variation of activation energy with alloy composition can be understood on the basis of Miedema's (1979) semi-empirical approach for vacancy formation in metals and alloys.

Valence band and core level spectroscopy of these glasses, using electron spectroscopy for chemical analysis (ESCA), shows a significant modification in the band shapes and positions. ESCA valence band spectrum of copper in the alloys shifts by nearly $0.8 \mathrm{eV}$ towards higher binding energies whereas $\mathrm{Zr} d$ band moves towards the Fermi level. These results differ from those for single phase alloys like $\mathrm{Cu}-\mathrm{Ni}$ and $\mathrm{Cu}-\mathrm{Pt}$ where no relative shift of constituent $d$ resonances has been observed. A detailed analysis of photoemission results of the constituent elements, glasses and their crystalline forms in terms of valence band calculations and soft x-ray emission spectroscopy results has been carried out and is described elsewhere (Budhani et al 1981b).

Since copper $d$ resonance in the alloy is far from the Fermi level, the electron transport behaviour of the alloys is primarily determined by $\mathrm{Zr} d$ contribution and by $s-p$ electrons. Conduction mechanism in general can be understood in terms of Evans et al (1971) approach for electron conduction in transition metal liquids.

Unlike $\mathrm{Cu}-\mathrm{Zr}$ type pure transition metal-based alloys, $\mathrm{Pd}-\mathrm{Ge}$ is a metal metalloid type glass with a very narrow glass forming range confined around the eutectic composition $(20 \% \mathrm{Ge})$. Melt-spinning of these alloys leads to glass formation from 20 to $22 \cdot 5 \% \mathrm{Ge}$. Detailed electron microscopy and x-ray diffraction studies on as-quenched and annealed glasses reveal that the structure transforms to equilibrium alloy $\alpha-\mathrm{Pd}$ and $\mathrm{Pd}_{3} \mathrm{Ge}$ through eutectic reaction. The activation energy for the transformation process as calculated from resistivity behaviour is $2 \cdot 78 \mathrm{eV}$.

Some structural (Polk 1972) and electronic models (Negel and Tauc 1975) speculate that an ionic bonding and a decrease in the density of states at $E_{F}$ is necessary for stability of the glassy phase. Our detailed photoemission and Auger electron spectroscopy studies (Budhani et al 1981c) do not indicate a charge transfer from $\mathrm{Ge}$ to $\mathrm{Pd}$ which could have led to ionic bonding in the glass. Density of states at $E_{\mathrm{F}}$ is, however, significantly modified. In elemental palladium and the crystalline alloy, $\mathrm{Pd} d$ band is close to the Fermi level and the density of states at $E_{F}\left[N\left(E_{F}\right)\right]$ is large. Large $N\left(E_{z}\right)$ is also reflected in the shape of the core levels. For the glass alloy, XPS studies show a narrow $d$ band located at $2 \mathrm{eV}$ below $E_{F}$. Valence band spectra and shapes of the core levels indicate that the nature of states at $E_{F}$ is $s-p$ type. Low $N\left(E_{F}\right)$ is also reflected in electrical resistivity and thermoelectric power measurements for the glass.

Finally, we must point out that amorphous metallic and semiconducting structures can also be prepared by vapour-quenching processes (Chopra 1969). The most attractive feature of the vapour quenching and sputter quenching is the associated high cooling rate which is in the range of $10^{12} \sim 10^{14}{ }^{\circ} \mathrm{K} / \mathrm{sec}$. Akhtar et al (1979b) in a detailed comparative study of liquid and vapour quenching techniques have shown that the latter leads to more disordered and metastable 
phase formation. Several non-crystalline metal semiconductor alloy systems $\mathrm{M}_{X} \mathrm{Gs}_{1-x}$ (Randhawa et al 1976), where $M$ stands for metals and metal-metal systems like $\mathrm{Cu}-\mathrm{Ag}$ (Thakoor 1975) have been prepared in our laboratory by thermal evaporation techniques. In sputter-quenching, an additional feature which enhances the cooling rate is the initial kinetic energy of the atoms. The work of Chopra et al (1967) on sputter deposition of $\mathrm{Zr}, \mathrm{W}$, Mo, etc., films has shown that this process leads to a variety of metastable and amorphous phases.

\section{Concluding remarks}

In view of the tremendous growth in the field of rapid solidification and variety of applications where rapidly solidified materials can be used, a detailed account of the physical and engineering aspects of these materials would certainly be a voluminous task. A number of reviews (Guntherodt et al 1979 ; Cahn 1980 ; Cargill 1975; Anantharaman et al 1977), topical articles (Chaudhari et al 1980; Gilman 1975) and proceedings of international conferences (Grant and Giessen 1976 ; Cantor 1978), dealing with many facets of the process, have appeared. Finer details of a particular technique application can be obtained therein.

\section{Acknowledgement}

The authors would like to thank Dr T V Rao for his cooperation in the development of the vacuum system.

\section{References}

Akhtar D, Vankar V D, Gael T C and Chopra K L 1979a J. Mater. Sci. 14983

Akhtar D, Goel T C, Vankar V D and Chopra K L 1979b Thin Solid Films 58327

Anantharaman T, Ramachandrarao P, Suryanarayana C, Lele S and Chattopadhyay 1977 Trans. IIM Rev. No. 6

Anthony T R and Cline H E 1979 J. Appl. Phys. 50245

Berkowitz A E, Walter J L and Wall K F 1981 Phys. Rev. Lett. 461484

Budhani R C, Goel T C and Chopra K L 1981a Presented at 4th Int. Conf. on Rapidly Quenched Metals, Sendai, Japan

Budhani R C, Goel T C and Chapra K L $1981 b$ to be communicated.

Budhani R C, Rajagopalan S, Banerjee A, Goel T C and Chopra K L 1981c Presented at 5th Int. Congr. on Thin Films, Israel.

Cahn R W 1980 Contemp. Phys. 2143

Cantor B 1978 in Rapidly quenched metals III (London : Chameleon Press)

Cargill G S 1975 in Solid state physics Vol 25 (eds.) H Ehrenreich, F Seitz and D Turnbul (New York : Academic Press)

Chaudhari P, Giessen B C and Turnbull D 1980 Sci. Am. 24298

Chen H S and Miller C E 1970 Rev. Sci. Instrum. 411237

Chopra K L 1969 Thin film phenomena (New York: McGraw-Hill)

Chopra K L, Randlett M R and Duff R H 1967 Philos. Mag. 16261

Clampitt R, Scott M G, Aitken K L, Lawland L and Prewett P D 1978 Proceedings III Int. Conf. on Rapidly quenched metals, (ed.) B Cantor (London : Chameleon Press Ltd.) p. 57

Cline H E and Anthony T R 1978 General Electric Corporation R and D Lab. Report No. 78 CRD 118 
Davis H A 1978 in Proceedings of III Int. Conf. on Rapidly quenched metals (ed.) B Cantor (London: Chameleon Press Ltd.) p. 1

Duwez P and Willens R H 1963 Trans. Met. Soc. AlME 227362

Evans R, Greenwood D A and Lloyd P 1971 Phys. Lett. A35 57

Giessen B C, Madhava N M, Murphy R J, Ray R and Surette J 1977 Met. Trans. A8 364

Gilman J J 1975 Phys. Today 2846

Grant N J and Giessen B C 1976 (Mass.: M.I.T. Press) Section I Section II-Mater. Sci. Eng. 197623 Section II/III.

Guntherodt $H$ J, Beck H, Oelhafen P, Ackermann K P, Liard M, Muller M, Künzi $H$ U, Rudin H and Agyeman K 1979 in Electrons in disordered metals and at metallic surfaces (eds.) P Phariseau, B L Gyorffy and L Scheire (New York: Plenum Press) p. 501

Johnson W L and Williams A R 1979 Phys. Rev. B20 1640

Liebermann H H and Graham C D 1976 IEEE Trans. Magn. Magn. Mater. Mag. 12921

Luborsky F E, Bocker J J, Frischman P G and Johnson L A 1978 J. Appl. Phys. 491769

Maringer R E and Mabley C E 1974 J. Vac. Sci. Tech. 111067

Miedema A R 1979 Z. Metallkd. 70345

Mizzlodi P, Della Mea G, Battaglin G, Miotello A, Servidori M, Bacci D and Janmitti E 1980 Phys. Rev. Lett. 4488

Negel S R and Tauc J 1975 Phys. Rev. Lett. 35380

Naka M, Hasimato $\mathrm{K}$ and Masumoto T $1978 \mathrm{~J}$. Non-Cryst. Solids 3029

Ohring $M$ and Haldipur A 1972 Rev. Sci. Instrum. 42530

Pietrakowsky P 1963 Rev. Sci. Instrum. 34445

Polk D 1972 Acta Metall. 20485

Pond R Jr and Maddin R 1969 Trans. Met. Soc. AIME 2452475

Rondhawa H S, Malhotra L K and Chopra K L 1916 Solid State Commun. 2073

Ruhl R C 1967 Mater. Sci. Eng. 1313

Suryanarayana C 1981 Rapidly ouenched metals-a bibliography (New York : Plenum)

Thakoor A P 1975 Ph.D. Thesis, Disorder Effects on Thermopower of Copper Alloy Films IIT, Delhi (unpublished).

Turnbull D 1969 Contemp. Phys. 10473

Walter J L and Lukborsky F E 1977 General Electric Corporation (USA) R and D Lab. Report 